\title{
Scalable Quantum Computing with Josephson Charge Qubits
}

\author{
J. Q. You, ${ }^{1}$ J. S. Tsai, ${ }^{1,2}$ and Franco Nori ${ }^{1,3, *}$ \\ ${ }^{1}$ Frontier Research System, The Institute of Physical and Chemical Research (RIKEN), Wako-shi 351-0198, Japan \\ ${ }^{2}$ NEC Fundamental Research Laboratories, Tsukuba, Ibaraki 305-8051, Japan \\ ${ }^{3}$ Center for Theoretical Physics, Physics Department, Center for the Study of Complex Systems, The University of Michigan, \\ Ann Arbor, Michigan 48109-1120 \\ (Received 11 March 2002; published 21 October 2002)
}

\begin{abstract}
A goal of quantum information technology is to control the quantum state of a system, including its preparation, manipulation, and measurement. However, scalability to many qubits and controlled connectivity between any selected qubits are two of the major stumbling blocks to achieve quantum computing (QC). Here we propose an experimental method, using Josephson charge qubits, to efficiently solve these two central problems. The proposed QC architecture is scalable since any two charge qubits can be effectively coupled by an experimentally accessible inductance. More importantly, we formulate an efficient and realizable QC scheme that requires only one (instead of two or more) two-bit operation to implement conditional gates.
\end{abstract}

PACS numbers: 03.67.Lx, 74.50.+r, 85.25.Cp

The macroscopic quantum effects in low-capacitance Josephson-junction circuits have recently been used to realize qubits for quantum information processing, and these qubits are expected to be scalable to large-scale circuits using modern microfabrication techniques. Josephson-qubit devices [1] are based on the charge and phase degrees of freedom. The charge qubit is achieved in a Cooper-pair box [2], where two dominant charge states are coupled through coherent Cooper-pair tunneling [3], while the phase qubit is based on two different flux states in a small superconducting-quantum-interference-device (SQUID) loop [4,5]. Experimentally, the energy-level splitting and the related properties of state superpositions were observed via Cooper-pair tunneling in the Josephson charge device [6,7] and by spectroscopic measurements for the Josephson phase device [8,9]. Moreover, coherent oscillations were demonstrated in a Josephson charge device prepared in a superposition of two charge states [2]. These striking experimental observations reveal that the Josephson charge and phase devices are suitable for solid-state qubits in quantum information processing. The next immediate challenge would include implementing a two-bit coupling and then scaling up the architecture to many qubits. Here, we focus on the Josephson charge qubit realized in a Cooper-pair box and propose a new quantum-computing (QC) scheme based on scalable charge-qubit structures.

A straightforward way of coupling Josephson charge qubits is to use the Coulomb interactions between charges on different islands of the charge qubits (e.g., to connect two Cooper-pair boxes via a capacitor). A two-bit operation [10], similar to the controlled-NOT gate, was derived using this interbit coupling, but it is hard to switch the coupling on and off [1] in this scheme as well as to make the system scalable because only neighboring qubits can be coupled. A scalable way of coupling Josephson charge qubits was proposed $[1,3]$ in terms of the oscillator modes in an $L C$ circuit formed by an inductance and the qubit capacitors. In this design, the interbit coupling is switchable and any two charge qubits can be coupled. However, there is no efficient (i.e., using one two-bit operation) QC scheme for this design [1,3] to achieve conditional gates such as the controlled-phase-shift and controlled-NOT gates. Moreover, the calculated interbit coupling terms $[1,3]$ apply only to the case when two conditions are met: (i) the eigenfrequency $\omega_{L C}$ of the $L C$ circuit is much faster than the quantum manipulation frequencies (which limits the allowed number $N$ of the qubits in the circuit because $\omega_{L C}$ scales with $1 / \sqrt{N}$ ) and (ii) the phase conjugate to the total charge on the qubit capacitors fluctuates weakly. These two limitations do not apply to our approach. In our proposal, a common inductance (but no $L C$ circuit) is used to couple all Josephson charge qubits. In our scheme, both dc and ac supercurrents can flow through the inductance, while in [1,3] only ac supercurrents can flow through the inductance and it is the $L C$-oscillator mode that couples the charge qubits. These yield different interbit couplings (e.g., $\sigma_{y} \sigma_{y}$ type $[1,3]$ as opposed to $\sigma_{x} \sigma_{x}$ in our scheme). To have a controllable interbit coupling, we employ two dc SQUIDs to connect each Cooper-pair box. Our proposed QC architecture is scalable in the sense that any two charge qubits (not necessarily neighbors) can be effectively coupled by an experimentally accessible inductance. More importantly, we formulate an efficient QC scheme that requires only one (instead of two or more) two-bit operation to implement conditional gates. To our knowledge, this is the first efficient scalable QC scheme for this type of architecture.

The proposed quantum computer consists of $N$ Cooperpair boxes coupled by a common superconducting inductance $L$ (see Fig. 1). For the $k$ th Cooper-pair box, a superconducting island with charge $Q_{k}=2 e n_{k}$ is weakly coupled by two symmetric dc SQUIDs and biased by an 


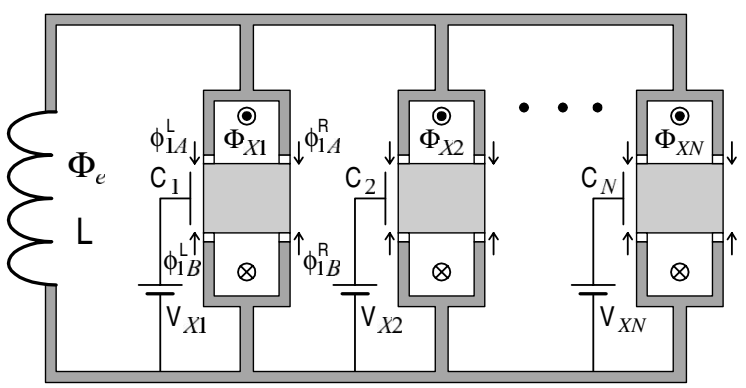

FIG. 1. Schematic diagram of the proposed scalable and switchable quantum computer. All Josephson charge-qubit structures are coupled by a common superconducting inductance. Here, each Cooper-pair box is operated both in the charging regime $E_{c k} \gg E_{J k}^{0}$ and at low temperatures $k_{B} T \ll$ $E_{c k}$. Moreover, the superconducting gap is larger than $E_{c k}$, so that quasiparticle tunneling is prohibited in the system.

applied voltage $V_{X k}$ through a gate capacitance $C_{k}$. The two symmetric de SQUIDs are assumed to be identical and all Josephson junctions in them have Josephson coupling energy $E_{J k}^{0}$ and capacitance $C_{J k}$. Each SQUID pierced by a magnetic flux $\Phi_{X k}$ provides an effective coupling energy given by $-E_{J k}\left(\Phi_{X k}\right) \cos \phi_{k A(B)}$, with $E_{J k}\left(\Phi_{X k}\right)=2 E_{J k}^{0} \cos \left(\pi \Phi_{X k} / \Phi_{0}\right)$, and $\Phi_{0}=h / 2 e$ is the flux quantum. The effective phase drop $\phi_{k A(B)}$, with subscript $A(B)$ labeling the SQUID above (below) the island, equals the average value, $\left[\phi_{k A(B)}^{L}+\phi_{k A(B)}^{R}\right] / 2$, of the phase drops across the two Josephson junctions in the dc SQUID, where the superscript $L(R)$ denotes the left (right) Josephson junction. Since the size of the loop is usually very small $(\sim 1 \mu \mathrm{m})$, above we have ignored the self-inductance effects of each SQUID loop. The Hamiltonian of the system is $H=\sum_{k=1}^{N} H_{k}+\frac{1}{2} L I^{2}$, with $H_{k}$ given by $H_{k}=E_{c k}\left(n_{k}-n_{X k}\right)^{2}-E_{J k}\left(\Phi_{X k}\right) \times$ $\left(\cos \phi_{k A}+\cos \phi_{k B}\right)$. Here, $E_{c k}=2 e^{2} /\left(C_{k}+4 C_{J k}\right)$ is the charging energy of the superconducting island and $I=$ $\sum_{k=1}^{N} I_{k}$ is the total persistent current through the superconducting inductance, as contributed by all coupled Cooper-pair boxes. The offset charge $2 e n_{X k}=C_{k} V_{X k}$ is induced by the gate voltage $V_{X k}$. The phase drops $\phi_{k A}^{L}$ and $\phi_{k B}^{L}$ are related to the total flux $\Phi=\Phi_{e}+L I$ through the inductance $L$ by the constraint $\phi_{k B}^{L}-\phi_{k A}^{L}=$ $2 \pi \Phi / \Phi_{0}$, where $\Phi_{e}$ is the externally applied magnetic flux threading the inductance $L$. Without loss of generality and in order to implement QC more conveniently, the magnetic fluxes through the two SQUID loops of each Cooper-pair box are designed to have the same values but opposite directions; this simplifies the form of the Hamiltonian. (If this were not to be the case, the interbit coupling can still be realized, but the Hamiltonian of the qubit circuits takes a more complicated form.) Because this pair of fluxes cancels each other in any loop enclosing them, then $\phi_{k B}^{L}-\phi_{k A}^{L}=\phi_{k B}^{R}-\phi_{k A}^{R}$. This gives rise to the constraint $\phi_{k B}-\phi_{k A}=2 \pi \Phi / \Phi_{0}$ for the average phase drops across the Josephson junctions in the SQUIDs. The common superconducting inductance $L$ plays the role of coupling Cooper-pair boxes. The coupling of selected Cooper-pair boxes can be implemented by switching on the SQUIDs connected to the chosen Cooper-pair boxes, and the persistent currents through the inductance $L$ are composed of contributions from all the coupled Cooper-pair boxes.

One-and two-bit circuits. - For any given Cooper-pair box, say $i$, when $\Phi_{X k}=\frac{1}{2} \Phi_{0}$ and $V_{X k}=\left(2 n_{k}+1\right) e / C_{k}$ for all boxes except $k=i$, the inductance $L$ connects only the $i$ th Cooper-pair box to form a superconducting loop [see Fig. 2(a)]. The Hamiltonian of the system can be reduced to [11]

$$
H=\varepsilon_{i}\left(V_{X i}\right) \sigma_{z}^{(i)}-\bar{E}_{J i}\left(\Phi_{X i}, \Phi_{e}, L\right) \sigma_{x}^{(i)},
$$

where $\varepsilon_{k}\left(V_{X k}\right)$ is controllable via the gate voltage $V_{X i}$, while the intrabit coupling $\bar{E}_{J k}\left(\Phi_{X i}, \Phi_{e}, L\right)$ can be controlled by both the applied external flux $\Phi_{e}$ through the common inductance, and the local flux $\Phi_{X i}$ through the two SQUID loops of the $i$ th Cooper-pair box. The intrabit coupling $\bar{E}_{J i}$ in (1) is different from that in $[1,3]$ because a very different contribution by $L$ is considered. To couple any two Cooper-pair boxes, say $i$ and $j$, we choose $\Phi_{X k}=$ $\frac{1}{2} \Phi_{0}$ and $V_{X k}=\left(2 n_{k}+1\right) e / C_{k}$ for all boxes except $k=i$ and $j$. As shown in Fig. 2(b), the inductance $L$ is shared by the Cooper-pair boxes $i$ and $j$ to form superconducting loops. The reduced Hamiltonian of the system is given by [13]

$$
H=\sum_{k=i, j}\left[\varepsilon_{k}\left(V_{X k}\right) \sigma_{z}^{(k)}-\bar{E}_{J k} \sigma_{x}^{(k)}\right]+\Pi_{i j} \sigma_{x}^{(i)} \sigma_{x}^{(j)} .
$$

Here the interbit coupling $\Pi_{i j}$ is controlled by both the external flux $\Phi_{e}$ through the inductance $L$, and the local fluxes, $\Phi_{X i}$ and $\Phi_{X j}$, through the SQUID loops.

Quantum computing.-The quantum system evolves according to $U(t)=\exp (-i H t / \hbar)$. Initially, we choose $\Phi_{X k}=\frac{1}{2} \Phi_{0}$ and $V_{X k}=\left(2 n_{k}+1\right) e / C_{k}$ for all boxes in Fig. 1, so that the Hamiltonian of the system is $H=0$ and no time evolution occurs. Afterwards, we switch certain fluxes $\Phi_{X k}$ and/or gate voltages $V_{X k}$ away from the above initial values for certain periods of times, to implement logic gates required for QC. For any two Cooper-pair boxes, say $i$ and $j$, when fluxes $\Phi_{X i}$ and $\Phi_{X j}$ are switched away from the initial value $\Phi_{0} / 2$ for a given period of time $\tau$, the Hamiltonian of the system becomes $H=-\bar{E}_{J i} \sigma_{x}^{(i)}-\bar{E}_{J j} \sigma_{x}^{(j)}+\Pi_{i j} \sigma_{x}^{(i)} \sigma_{x}^{(j)}$. This anisotropic Hamiltonian is Ising-like [14], with its anisotropic direction and the "magnetic" field along the $x$ axis. When the parameters are suitably chosen so that $\bar{E}_{J i}=$ $\bar{E}_{J j}=\Pi_{i j}=-\pi \hbar / 4 \tau$ for the switching time $\tau$, we obtain a controlled-phase-shift gate, $U_{\mathrm{CPS}}^{\prime}=e^{i \pi / 4} U_{2 b}=$ $\exp \left\{i \frac{\pi}{4}\left[1-\sigma_{x}^{(i)}-\sigma_{x}^{(j)}+\sigma_{x}^{(i)} \sigma_{x}^{(j)}\right]\right\}$, which does not alter the two-bit states $|+\rangle_{i}|+\rangle_{j},|+\rangle_{i}|-\rangle_{j}$, and $|-\rangle_{i}|+\rangle_{j}$ but transforms $|-\rangle_{i}|-\rangle_{j}$ to $-|-\rangle_{i}|-\rangle_{j}$. Here, the phase factor $e^{i \pi / 4}$ corresponds to an overall energy shift of the Hamiltonian, and $| \pm\rangle$ are defined by $| \pm\rangle=(|\uparrow\rangle \pm|\downarrow\rangle) / \sqrt{2}$.

To obtain the controlled-phase-shift gate $U_{\text {CPS }}$ for the basis states $|\uparrow\rangle_{i}|\uparrow\rangle_{j},|\uparrow\rangle_{i}|\downarrow\rangle_{j},|\downarrow\rangle_{i}|\uparrow\rangle_{j}$, and $|\downarrow\rangle_{i}|\downarrow\rangle_{j}$, one 
(a)

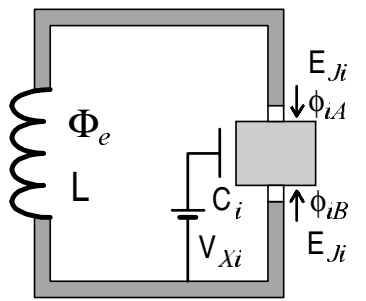

(b)

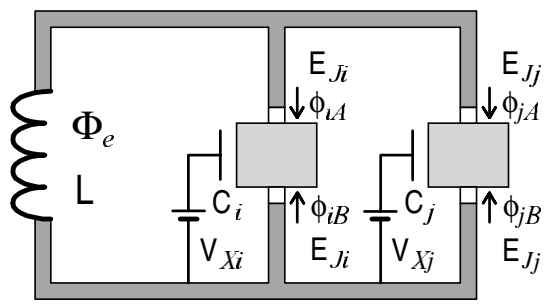

FIG. 2. (a) One-bit circuit with a Cooper-pair box connected to the inductance. (b) Two-bit structure where two Cooper-pair boxes are commonly connected to the inductance. Here, each SQUID connecting the superconducting island is represented by an effective Josephson junction.

needs to combine $U_{\mathrm{CPS}}^{\prime}$ with suitable one-bit rotations. For any Cooper-pair box, say $i$, one can shift flux $\Phi_{X i}$ and/or gate voltage $V_{X i}$ for a given switching time $\tau$ to derive one-bit rotations. A universal set of onebit gates $U_{z}^{(i)}(\alpha)=e^{i \alpha \sigma_{z}^{(i)}}$, and $U_{x}^{(i)}(\beta)=e^{i \beta \sigma_{x}^{(i)}}$, where $\alpha=-\varepsilon_{i}\left(V_{X i}\right) \tau / \hbar$ and $\beta=\bar{E}_{J i} \tau / \hbar$, can be defined by choosing $\bar{E}_{J i}=0$ and $\varepsilon_{i}\left(V_{X i}\right)=0$ (which can be done with suitable choices of $\Phi_{X i}$ and $\left.V_{X i}\right)$ in the one-bit Hamiltonian (1), respectively. Any one-bit rotation can be derived in terms of these two types of one-bit gates. For instance, the Hadamard gate is given by $\mathcal{H}_{i}=e^{-i \pi / 2} U_{z}^{(i)}\left(\frac{\pi}{4}\right) U_{x}^{(i)}\left(\frac{\pi}{4}\right) U_{z}^{(i)}\left(\frac{\pi}{4}\right)$. Using $\mathcal{H}_{i}$, we derive the controlled-phase-shift gate $U_{\mathrm{CPS}}$ : $U_{\mathrm{CPS}}=$ $\mathcal{H}_{j}^{\dagger} \mathcal{H}_{i}^{\dagger} U_{\mathrm{CPS}}^{\prime} \mathcal{H}_{i} \mathcal{H}_{j}$. The one-bit rotation $V_{j}=e^{i \pi \sigma_{y}^{(j)} / 4}$ is given by $V_{j}=U_{z}^{(j)}\left(-\frac{\pi}{4}\right) U_{x}^{(j)}\left(\frac{\pi}{4}\right) U_{z}^{(j)}\left(\frac{\pi}{4}\right)$. Combining $V_{j}$ with $U_{\mathrm{CPS}}$, we obtain the controlled-NOT gate, $U_{\mathrm{CNOT}}=V_{j}^{\dagger} U_{\mathrm{CPS}} V_{j}$, which transforms the basis states as $\quad|\uparrow\rangle_{i}|\uparrow\rangle_{j} \rightarrow|\uparrow\rangle_{i}|\uparrow\rangle_{j}, \quad|\uparrow\rangle_{i}|\downarrow\rangle_{j} \rightarrow|\uparrow\rangle_{i}|\downarrow\rangle_{j}, \quad|\downarrow\rangle_{i}|\uparrow\rangle_{j} \rightarrow$ $|\downarrow\rangle_{i}|\downarrow\rangle_{j}$, and $|\downarrow\rangle_{i}|\downarrow\rangle_{j} \rightarrow|\downarrow\rangle_{i}|\uparrow\rangle_{j}$. A sequence of such conditional two-bit gates supplemented with one-bit rotations constitute a universal element for QC [15]. Usually, a twobit operation is much slower than a one-bit operation. Our designs for conditional gates $U_{\mathrm{CPS}}$ and $U_{\mathrm{CNOT}}$ are efficient since only one (instead of two or more) two-bit operation $U_{\text {CPS }}^{\prime}$ is used.

Persistent currents and entanglement. - The one-bit circuit modeled by Hamiltonian (1) has two eigenvalues $E_{ \pm}^{(i)}= \pm E_{i}$, with $E_{i}=\left[\varepsilon_{i}^{2}\left(V_{X i}\right)+\bar{E}_{J i}^{2}\right]^{1 / 2}$. The corresponding eigenstates are $\left|\psi_{+}^{(i)}\right\rangle=\cos \xi_{i}|\uparrow\rangle_{i}-\sin \xi_{i}|\downarrow\rangle_{i}$, and $\quad\left|\psi_{-}^{(i)}\right\rangle=\sin \xi_{i}|\uparrow\rangle_{i}+\cos \xi_{i}|\downarrow\rangle_{i}$, where $\quad \xi_{i}=$ $\frac{1}{2} \tan ^{-1}\left(\bar{E}_{J i} / \varepsilon_{i}\right)$. At these two eigenstates, the persistent currents through the inductance $L$ are given by $\left\langle\psi_{ \pm}^{(i)}|I| \psi_{ \pm}^{(i)}\right\rangle= \pm\left(\bar{E}_{J i} I_{c i} / E_{i}\right) \sin \left(\pi \Phi_{e} / \Phi_{0}\right)+$ $\left(\pi L I_{c i}^{2} / 2 \Phi_{0}\right) \sin \left(2 \pi \Phi_{e} / \Phi_{0}\right)$, where the expansion in $I$ is retained up to the linear term in $\eta_{i}$. When a dc SQUID magnetometer is inductively coupled to the inductance $L$, these two supercurrents generate different fluxes through the SQUID loop of the magnetometer and the quantumstate information of the one-bit structure can be obtained from the measurements. To perform sensitive measurements with weak dephasing, one could use the underdamped dc SQUID magnetometer designed previously for the Josephson phase qubit $[4,8]$.
For the two-bit structure described by Eq. (2), the Hamiltonian has four eigenstates and the supercurrents through inductance $L$ take different values at these states. The fluxes produced by the supercurrents through $L$ can also be detected by the dc SQUID magnetometer. For instance, when $\varepsilon_{k}\left(V_{X k}\right)=0$ and $\bar{E}_{J k}>0$ for $k=i$ and $j$, the four eigenstates of the two-bit structure are

$$
\begin{aligned}
|1\rangle & =\frac{1}{2}\left(|\uparrow\rangle_{i}|\uparrow\rangle_{j}-|\uparrow\rangle_{i}|\downarrow\rangle_{j}-|\downarrow\rangle_{i}|\uparrow\rangle_{j}+|\downarrow\rangle_{i}|\downarrow\rangle_{j}\right), \\
|2\rangle & =\frac{1}{2}\left(|\uparrow\rangle_{i}|\uparrow\rangle_{j}+|\uparrow\rangle_{i}|\downarrow\rangle_{j}-|\downarrow\rangle_{i}|\uparrow\rangle_{j}-|\downarrow\rangle_{i}|\downarrow\rangle_{j}\right), \\
|3\rangle & =\frac{1}{2}\left(|\uparrow\rangle_{i}|\uparrow\rangle_{j}-|\uparrow\rangle_{i}|\downarrow\rangle_{j}+|\downarrow\rangle_{i}|\uparrow\rangle_{j}-|\downarrow\rangle_{i}|\downarrow\rangle_{j}\right), \\
|4\rangle & =\frac{1}{2}\left(|\uparrow\rangle_{i}|\uparrow\rangle_{j}+|\uparrow\rangle_{i}|\downarrow\rangle_{j}+|\downarrow\rangle_{i}|\uparrow\rangle_{j}+|\downarrow\rangle_{i}|\downarrow\rangle_{j}\right) .
\end{aligned}
$$

When expansions in $I_{i}$ and $I_{j}$ are retained up to the linear terms in $\eta_{i}$ and $\eta_{j}$, the corresponding supercurrents through inductance $L$ are $\langle k|I| k\rangle=I_{k} \sin \left(\pi \Phi_{e} / \Phi_{0}\right)+$ $\left(\pi L I_{k}^{2} / 2 \Phi_{0}\right) \sin \left(2 \pi \Phi_{e} / \Phi_{0}\right)$ for $k=1$ to 4 , where $I_{1}=$ $-\left(I_{c i}+I_{c j}\right), \quad I_{2}=I_{c j}-I_{c i}, \quad I_{3}=I_{c i}-I_{c j}$, and $I_{4}=$ $I_{c i}+I_{c j}$. These supercurrents produce different fluxes threading the SQUID loop of the magnetometer and can be distinguished by dc SQUID measurements. If the two-bit system is prepared at the maximally entangled Bell states $\left|\Psi^{( \pm)}\right\rangle=\left(|\uparrow\rangle_{i}|\downarrow\rangle_{j} \pm|\downarrow\rangle_{i}|\uparrow\rangle_{j}\right) / \sqrt{2}$, the supercurrents through $L$ are given by $\left\langle\Psi^{( \pm)}|I| \Psi^{( \pm)}\right\rangle=$ $\left(\pi L / 2 \Phi_{0}\right)\left(I_{c i} \pm I_{c j}\right)^{2} \sin \left(2 \pi \Phi_{e} / \Phi_{0}\right)$. These two states should be distinguishable by detecting the fluxes (generated by the supercurrents) through the SQUID loop of the magnetometer.

Discussion. - The typical switching time $\tau^{(1)}$ during a one-bit operation is of the order $\hbar / E_{J}^{0}$. For the experimental value of $E_{J}^{0} \sim 100 \mathrm{mK}$, there is $\tau^{(1)} \sim 0.1 \mathrm{~ns}$. The switching time $\tau^{(2)}$ for the two-bit operation is typically of the order $(\hbar / L)\left(\Phi_{0} / \pi E_{J}^{0}\right)^{2}$. Choosing $E_{J}^{0} \sim 100 \mathrm{mK}$ and $\tau^{(2)} \sim 10 \tau^{(1)}$ (i.e., 10 times slower than the one-bit rotation), we have $L \sim 30 \mathrm{nH}$ in our proposal, which is experimentally accessible. A small-size inductance with this value can be made with Josephson junctions. Our expansion parameter $\eta$ is of the order $\pi^{2} L E_{J}^{0} / \Phi_{0}^{2} \sim 0.1$. Our inductance $L$ is related with the inductance $L^{\prime}$ in $[1,3]$ by $L^{\prime}=\left(C_{J} / C_{q b}\right)^{2} L$. Let us now consider the case when $\tau^{(2)} \sim 10 \tau^{(1)}$. For the earlier design [3], $C_{J} \sim 11 C_{q b}$ since $C_{g} / C_{J} \sim 0.1$, which requires the inductance $\sim 3.6 \mu \mathrm{H}$. Such a large inductance is difficult to fabricate at nanometer scales. In the improved design [1], $C_{J} \sim 2 C_{q b}$, 
greatly reducing the inductance to $\sim 120 \mathrm{nH}$. This inductance is about 4 times larger than the one used in our scheme.

All charge qubits suffer decoherence due to the fluctuations of voltage sources and fluxes. Reference [1] shows that the gate voltage fluctuations play the dominant role in producing decoherence. The estimated dephasing time is $\tau_{\varphi} \sim 10^{-4} \mathrm{~s}$, allowing in principle $10^{6}$ coherent singlebit manipulations. When a probe junction is used for measurements, the experimental observations of coherent oscillations in the Josephson charge qubits show that the phase coherence time is only about $2 \mathrm{~ns}[2,16]$. In this experimental setup, background charge fluctuations and the probe-junction measurement may be two of the major factors in producing decoherences. Though the charge fluctuations are important only in the low-frequency region and can be reduced by the echo technique [16] and by shifting the gate voltage to the degeneracy point, an effective technique for suppressing charge fluctuations still needs to be explored. As for the measurement, it has also been a challenge to design effective detecting devices.

In conclusion, we propose a scalable quantum computer with Josephson charge qubits. We employ a common inductance to couple all charge qubits and design switchable interbit couplings using two dc SQUIDs to connect the island in each Cooper-pair box. The proposed QC architectures are scalable since any two charge qubits can be effectively coupled by an experimentally accessible inductance. Furthermore, we formulate an efficient QC scheme in which only one two-bit operation is used in the conditional transformations such as controlled-phaseshift and controlled-NOT gates.

We thank Yu. Pashkin for useful discussions. We acknowledge support from the U.S. ARDA, AFOSR, and the U.S. National Science Foundation Grant No. EIA-0130383.

*Corresponding author.

Electronic address: nori@umich.edu

[1] See, e.g., Y. Makhlin, G. Schön, and A. Shnirman, Rev. Mod. Phys. 73, 357 (2001), and references therein.

[2] Y. Nakamura, Yu. A. Pashkin, and J.S. Tsai, Nature (London) 398, 786 (1999).

[3] Y. Makhlin, G. Schön, and A. Shnirman, Nature (London) 398, 305 (1999).

[4] J. E. Mooij et al., Science 285, 1036 (1999).

[5] T. P. Orlando et al., Phys. Rev. B 60, 15398 (1999).

[6] Y. Nakamura, C. D. Chen, and J. S. Tsai, Phys. Rev. Lett. 79, 2328 (1997).

[7] V. Bouchiat et al., Phys. Scr. T76, 165 (1998).

[8] C. H. van der Wal et al., Science 290, 773 (2000).

[9] J. R. Friedman et al., Nature (London) 406, 43 (2000).

[10] F. Plastina, R. Fazio, and G. M. Palma, Phys. Rev. B 64, 113306 (2001).
[11] The Hamiltonian of the one-bit circuit shown in Fig. 2(a) is $H=H_{i}+\frac{1}{2} L I_{i}^{2}$, with $H_{i}=E_{c i}\left(n_{i}-n_{X i}\right)^{2}-$ $2 E_{J i}\left(\Phi_{X i}\right) \cos \left(\pi \Phi / \Phi_{0}\right) \cos \varphi_{i}$. Here, the phase $\varphi_{i}=$ $\left(\phi_{i A}+\phi_{i B}\right) / 2$ is canonically conjugate with the number of the extra Cooper pairs on the island and the persistent circulating current $I_{i}$ in the superconducting loop is given by $I_{i}=2 I_{c i} \cos \varphi_{i} \sin \left(\pi \Phi_{e} / \Phi_{0}+\pi L I_{i} / \Phi_{0}\right)$, where $I_{c i}=-\pi E_{J i}\left(\Phi_{X i}\right) / \Phi_{0}$. In the spin- $\frac{1}{2}$ representation, based on charge states $|\uparrow\rangle_{i}=\left|n_{i}\right\rangle$ and $|\downarrow\rangle_{i}=\left|n_{i}+1\right\rangle$, the reduced Hamiltonian of the system becomes Eq. (1), where $\varepsilon_{i}\left(V_{X i}\right)=\frac{1}{2} E_{c i}\left[C_{i} V_{X i} / e-\left(2 n_{i}+1\right)\right]$, and $\bar{E}_{J i}\left(\Phi_{X i}, \Phi_{e}, L\right)=E_{J i}\left(\Phi_{X i}\right)\left(\gamma_{i}+\eta_{i} \alpha_{i} \beta_{i}\right)$. Here $\alpha_{i}, \beta_{i}$, and $\gamma_{i}$ are power series of the expansion parameter $\eta_{i}=\pi L I_{c i} / \Phi_{0}$, which is $\sim 0.1$ in our case (see the discussion part above). These have the same expressions as $\alpha, \beta$, and $\gamma$ in Ref. [12], with $I_{c}$ and $\Phi_{X}$ there replaced by $I_{c i}$ and $\Phi_{e}$. Retained up to secondorder terms in the expansion parameter, $\bar{E}_{J i}\left(\Phi_{X i}, \Phi_{e}, L\right)=E_{J i}\left(\Phi_{X i}\right) \cos \left(\pi \Phi_{e} / \Phi_{0}\right) \xi$, with $\xi=$ $1-\frac{1}{2} \eta_{i}^{2} \sin ^{2}\left(\pi \Phi_{e} / \Phi_{0}\right)$.

[12] J. Q. You, C.-H. Lam, and H. Z. Zheng, Phys. Rev. B 63, 180501(R) (2001).

[13] The Hamiltonian of the two-bit circuit given in Fig. 2(b) is $H=H_{i}+H_{j}+\frac{1}{2} L\left(I_{i}+I_{j}\right)^{2}$, where $I_{i}=$ $2 I_{c i} \cos \varphi_{i} \sin \left[\pi \Phi_{e} / \Phi_{0}+\pi L\left(I_{i}+I_{j}\right) / \Phi_{0}\right]$ is the persistent circulating current contributed by the Cooper-pair box $i$. Interchanging $i$ and $j$ in $I_{i}$ gives the circulating current $I_{j}$ contributed by the Cooper-pair box $j$. In the spin- $\frac{1}{2}$ representation, the Hamiltonian of the system reduces to Eq. (2), where the intrabit coupling $\bar{E}_{J k}$ and the interbit coupling $\Pi_{i j}$ are also power series, which have the same expressions as in Ref. [12], but with $L_{i}, L_{j}$, and $M_{i j}$ replaced by $L ; \Phi_{X i}$ and $\Phi_{X j}$ replaced by $\Phi_{e}$; and $E_{J k}^{0}$ replaced by $E_{J k}\left(\Phi_{X k}\right)$. Up to second-order terms, $\quad \bar{E}_{J i}\left(\Phi_{X i}, \Phi_{e}, L\right)=E_{J i}\left(\Phi_{X i}\right) \cos \left(\pi \Phi_{e} / \Phi_{0}\right) \xi$, with $\xi=1-\frac{1}{2}\left(\eta_{i}^{2}+3 \eta_{j}^{2}\right) \sin ^{2}\left(\pi \Phi_{e} / \Phi_{0}\right)$, and $\Pi_{i j}=$ $-L I_{c i} I_{c j} \sin ^{2}\left(\pi \Phi_{e} / \Phi_{0}\right)$. When the two qubits are far apart, the inductance of the wires connecting them might not be neglected. However, the reduced two-bit Hamiltonian is still given by Eq. (2), but with $\bar{E}_{J k}$ and $\Pi_{i j}$ slightly modified. In deriving the interbit coupling $\Pi_{i j}$, here we ignore the collective $L C$-oscillator mode associated with the total charge $q$ accumulated on the gate capacitors of the arrays of Cooper-pair boxes [1]. When it is taken into account, a term $(q-$ $\left.\sum_{k} 2 e n_{k} \chi_{k}\right)^{2} / 2 C_{\Sigma}$, with $C_{\Sigma}=\sum_{k} 2 C_{J}\left(C_{k}+2 C_{J}\right) /$ $\left(C_{k}+4 C_{J}\right)$ and $\chi_{k}=C_{k} /\left(C_{k}+4 C_{J}\right)$, needs to be included in the Hamiltonian. Fast collective oscillations in the $L C$ circuit then yield an effective coupling between qubits $i$ and $j$, and the effective coupling strength $\left|\Lambda_{i j}\right|$ is $\sim \chi_{i} \chi_{j}\left|\Pi_{i j}\right|$. For typical values of $C_{k} / C_{J} \sim 0.1$, there is $\chi_{k} \sim 0.025$. Thus, we have $\left|\Lambda_{i j}\right| \sim 6 \times$ $10^{-4}\left|\Pi_{i j}\right|$, implying that neglecting the $L C$-oscillator effect here is quite reasonable.

[14] G. Burkard et al., Phys. Rev. B 60, 11404 (1999).

[15] S. Lloyd, Phys. Rev. Lett. 75, 346 (1995); D. Deutsch, A. Barenco, and A. Ekert, Proc. R. Soc. London A 449, 669 (1995).

[16] Recent measurement shows that the oscillations survive up to $5 \mathrm{~ns}$; see Y. Nakamura, Yu. A. Pashkin, T. Yamamoto, and J. S. Tsai, Phys. Rev. Lett. 88, 047901 (2002). 